\title{
Valoración hedónica de la inseguridad en la determinación de precios de viviendas en Acapulco de Juárez, Guerrero*
}

\author{
Hedonic Valuation of the Insecurity in the Determination \\ of Prices of Housing in Acapulco de Juárez, Guerrero
}

\author{
Joaquín Delgado Fernández y Giovanni Wences Nájera ${ }^{* * *}$
}

\section{RESUMEN}

En este trabajo llevamos a cabo un estudio sobre el efecto de la inseguridad en los precios de viviendas en Acapulco de Juárez usando un modelo econométrico de precios hedónicos. Para ello es considerada en nuestro modelo la distancia de viviendas a la colonia más insegura de dicha ciudad como la variable que medirá tal impacto. Como en la literatura existente, en este modelo consideramos atributos estructurales de las viviendas, así también amenidades que tienen que ver con su localización, como la distancia a la playa. La mayor parte de los atributos considerados en este estudio resultaron ser significativos estadísticamente, en particular nuestra variable de más interés, cuyo coeficiente resulta ser positivo; así que podemos imputar una pérdida de valor de la vivienda cuando ésta se encuentra cerca de la colonia más insegura. Con este enfoque hedónico obtenemos cuantificaciones del impacto de estos factores en la determinación de precios de viviendas en Acapulco.

Palabras clave: Valuación de vivienda, regresión hedónica, Acapulco, inseguridad, precio implícito marginal, transformación Box-Cox.

Clasificación JEL: R3, R31.

\begin{abstract}
In this paper, we conducted a study on the effect of insecurity on housing prices in Acapulco de Juárez using an econometric model of hedonic prices. To do this, the distance of the most unsafe neighborhood in the city from the housing is considered as the variable that will measure such impact. As in the existing literature, in this model we consider also structural attributes of the housing, as well as amenities that have to do with their location, such as the distance to the beach. Most of the attributes considered in this study turned out to be statistically significant, particularly our most interesting variable, whose coefficient turns out to be positive; so we can impute a loss of value of the housing when it is near the most insecure neighborhood. With this hedonic approach we obtain quantifications of the impact of these factors in the determination of housing prices in Acapulco.
\end{abstract}

Keywords: Housing valuation, hedonic regression, Acapulco, insecurity, marginal implicit price, Box-Cox transformation.

JEL Classification: R3, R31.

\footnotetext{
${ }^{*}$ Fecha de recepción: 15/06/2017. Fecha de aceptación: 24/07/2018.

** Univesidad Autónoma Metropolitana, Unidad Iztapalapa. Correo: jdf@xanum.uam.mx. ORCID: 0000-0002-7316-6672.

*** Univesidad Autónoma Metropolitana, Unidad Iztapalapa. Correo: giovanni.wences@gmail.com. ORCID: 00000003-3745-2118.
} 


\section{INTRODUCCIÓN}

El puerto de Acapulco de Juárez, Guerrero, es uno de los puertos más atractivos de México que atrae tanto a turistas nacionales como extranjeros. La gran diversidad de playas, libres y privadas, sus centros nocturnos y la cercanía a la capital del país convierte a este puerto en uno de los más visitados. Acapulco ha sido uno de los más importantes en México debido a la contribución económica al estado de Guerrero. Por ejemplo, México ocupa el octavo lugar entre los países que más turismo reciben (World Tourism Organization, 2015), en contraste, Acapulco ocupa el primer lugar entre las ciudades del país con mayor índice de violencia, lo cual ha afectado a muchos sectores sociales en el puerto. De hecho, el Consejo Ciudadano para la Seguridad Pública y Justicia Penal declaró que durante los periodos 2015 y 2016, Acapulco fue la ciudad más violenta del país (Seguridad, Justicia y Paz, 2015; Seguridad, Justicia y Paz, 2016).

Con base en esta problemática, nuestro objetivo principal en este artículo es conocer a través de un modelo de precios hedónicos los efectos de la inseguridad en el valor de las viviendas en Acapulco de Juárez. Nuestras preguntas de investigación son:

1. ¿Cuáles son las variables que explican con mayor nivel de significancia el precio de las viviendas en Acapulco de Juárez?

2. ¿Cuál es el impacto cuantitativo de la inseguridad sobre el precio de las viviendas en Acapulco de Juárez?

3. Si se incluye la cercanía a la playa y lejanía de la colonia más insegura de una vivienda, como variables hedónicas, ¿cómo el comprador cuantifica estas variables en la determinación del precio de la vivienda?

En este trabajo evaluamos la hipótesis de que el efecto de la inseguridad, medido por la distancia de las viviendas a la colonia más insegura en Acapulco, es negativo sobre los precios de mercado de las viviendas en la ciudad de Acapulco, es decir, el valor de una vivienda disminuye con la cercanía a la colonia más insegura.

Para atender a los planteamientos anteriores aplicamos la teoría de los precios hedónicos para la valoración de atributos sobre viviendas en esta ciudad. En nuestro estudio proponemos un modelo que desagregue al precio en función de atributos que contribuyen al precio en el mercado de determinada vivienda. Algunos de estos atributos son típicos en la literatura de la aplicación empírica de la metodología hedónica en la valoración de viviendas (Baranzini, A., Ramírez, 
J., Schaerer, C. y Thalmann, P., 2008; Valdivia, R., 2014; Witte, A. D., Sumka, Howard J. y Erekson, H., 1979). Estos corresponden a atributos de carácter físico o estructural, tales como superficie construida, número de habitaciones o baños, etcétera. Otras variables consideradas tienen un carácter hedónico y tienen que ver con la percepción de amenidades o desamenidades que tienden a valorar o desvalorar el precio de un bien inmueble. Dos de estas características de viviendas consideradas en nuestro estudio, creemos que tienen un peso importante en la determinación del precio de una vivienda en mercado: $a$ ) la proximidad a la playa y $b$ ) la lejanía a zonas de alto índice delictivo. Incorporamos estos factores mediante variables de carácter geográfico tales como distancia en ruta a alguna avenida principal con acceso a la playa, y la distancia de la vivienda a la colonia Ciudad Renacimiento, considerada como la más peligrosa en el puerto de Acapulco, según la Comisión Nacional de Seguridad (CNS) (fuente citada por El Universal).

\section{ASPECTOS SOCIOECONÓMICOS Y GEOGRÁFICOS DE ACAPULCO}

Acapulco de Juárez es una ciudad y puerto del estado de Guerrero, cabecera municipal del municipio homónimo localizado en la costa sur de la República Mexicana. Se localiza a 304 kilómetros de distancia de la capital de México. Acapulco de Juárez fue el primer puerto turístico de México y el enlace entre los océanos Pacífico y Atlántico durante la Época virreinal (Enciclopedia Guerrerense, 2012). Actualmente es la ciudad más importante del estado de Guerrero debido a su actividad turística y es una de las zonas metropolitanas más grandes del país; de acuerdo con los resultados del Censo de Población y Vivienda, realizado por el Instituto de Estadística y Geografía (INEGI) en 2010, la ciudad de Acapulco es la más poblada del estado de Guerrero con 673,479 habitantes (véase figura 1. Área Geoestadística Municipal 120010001, 2010). A lo largo de su extenso litoral se encuentran un gran número de playas, balnearios, grutas y zonas arqueológicas, convirtiéndolo en un centro vacacional internacional.

Acapulco está dividido en tres grandes zonas turísticas: Acapulco Tradicional, Acapulco Dorado y Acapulco Diamante. El puerto, el centro de la ciudad, así como barrios antiguos se encuentran en Acapulco Tradicional, mientras que la parte con más afluencia de turistas se encuentra en Acapulco Dorado. Acapulco Diamante está conformado por hoteles, complejos residenciales, condominios exclusivos y con mayor desarrollo turístico. 
Figura 1. Crecimiento demográfico de la Ciudad de Acapulco de Juárez de 1900 a 2010. Los datos corresponden a los Censos de Población y Vivienda.

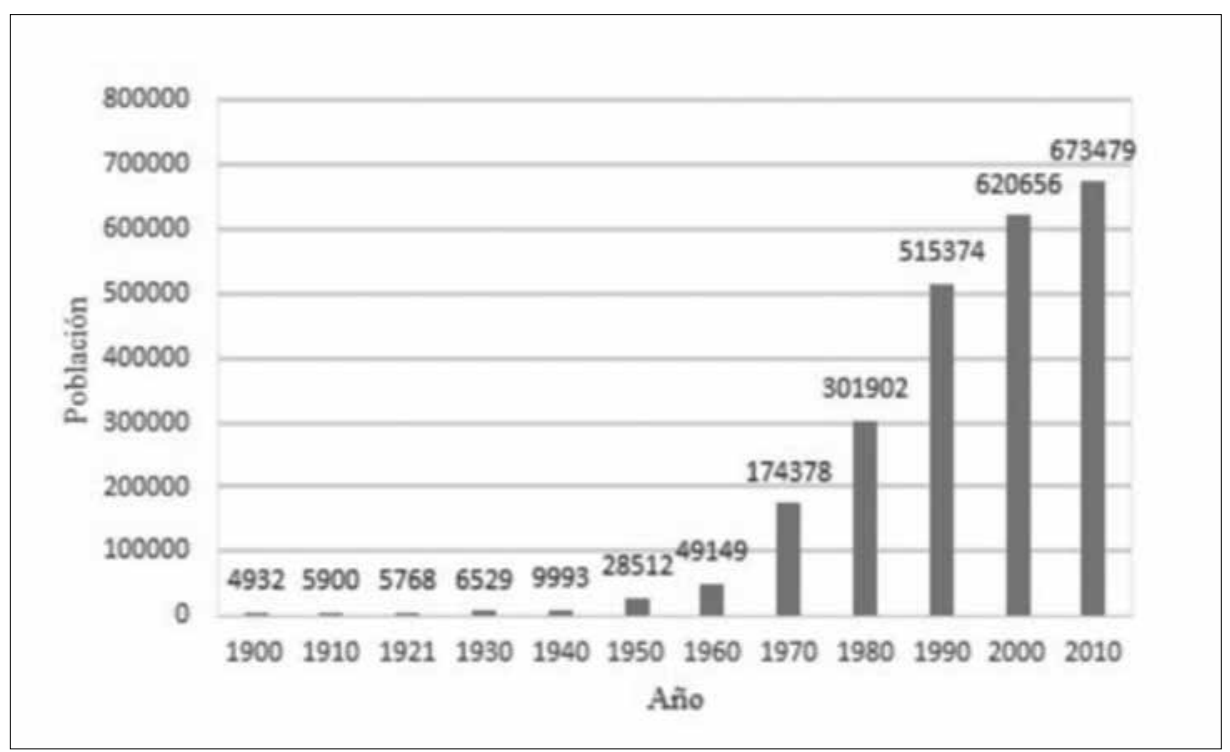

Fuente: Elaboración propia con datos del INEGI (Área Geoestadística Municipal 120010001, 2010).

La principal actividad económica de la ciudad es el turismo debido a su ingreso económico, sin embargo, debido a la ola violencia en los últimos años en esta ciudad, el turismo ha disminuido notablemente. Para los periodos 2014 y 2015, el Consejo Ciudadano para la Seguridad Pública y Justicia Penal, declaró a Acapulco como la ciudad más violenta de México debido al gran número de homicidios dolosos llevados a cabo en esos periodos (Seguridad, Justicia y Paz, 2015; Seguridad, Justicia y Paz, 2016).

Según la Comisión Nacional de Seguridad (CNS) la colonia más peligrosa de esta ciudad, debido al alto nivel de incidencia en homicidios dolosos, robo de vehículos y armamento asegurado, es la colonia Ciudad Renacimiento (fuente citada por El Universal, 2017).

Ciudad Renacimiento es una colonia ubicada en la zona periurbana de Acapulco de Juárez donde existen diferentes fuentes de contaminación, escasez de servicios, pobreza y sobrepoblación (López, Rodríguez, Barragán, Castellanos, Palacios y Martínez, 2012). La figura 2 muestra la extensión territorial, delimitada en oscuro claro, de la ciudad de Acapulco y la ubicación de la colonia Ciudad Renacimiento, delimitada por color negro. 
Figura 2. Ciudad de Acapulco de Juárez y localización de la colonia Ciudad Renacimiento.

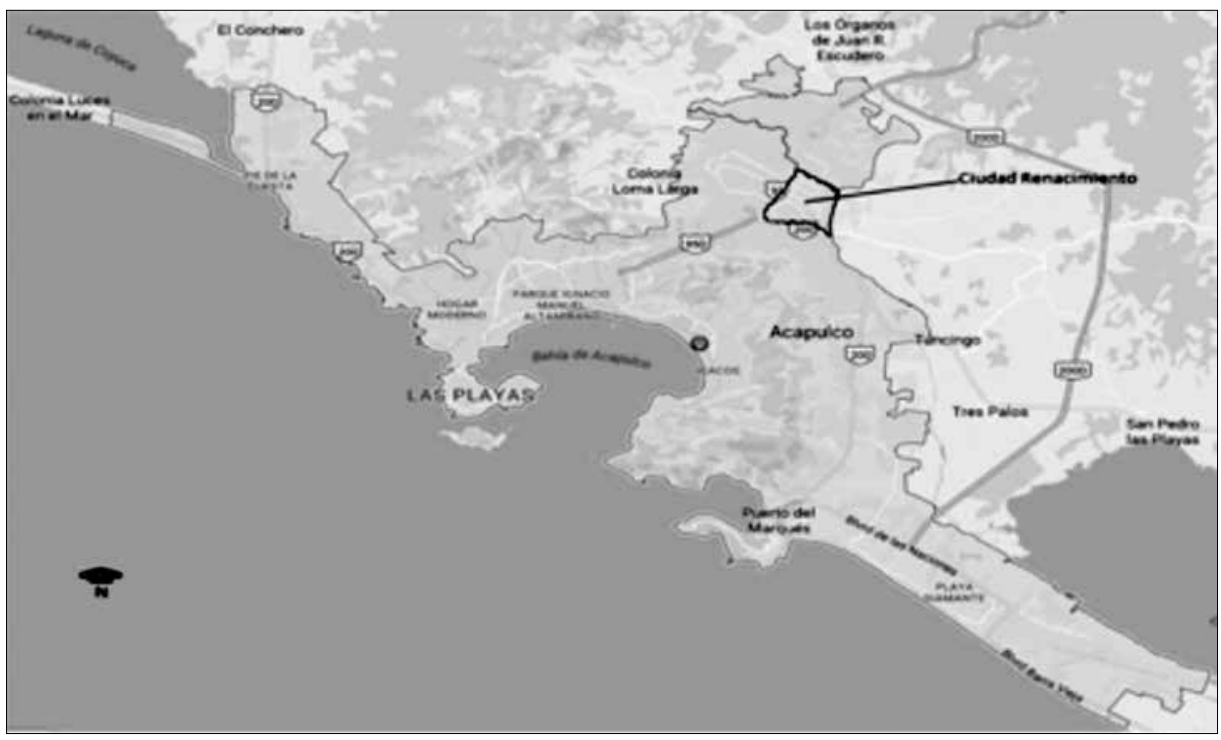

Fuente: Elaboración propia con Google Maps.

Canter (1977) define una vivienda como el lugar en el que además de los atributos físicos, se produce una relación entre éstos, las acciones que se dan en él y las concepciones que tienen las personas del mismo. Según Amérigo y Pérez-López (2010) la vivienda no sólo es un ambiente físico, sino que es un concepto cognitivo, afectivo y social. Así, cuando una persona compra una casa no sólo atiende a sus necesidades, sino también a sus emociones de placer. Acapulco es un lugar que por excelencia nos provoca un estado de placer por sus hermosas playas, sin embargo, desde que este puerto ha presentado altos índices de violencia, en los últimos años se percibe como un lugar que provoca temor. Este problema social ha afectado al mercado de viviendas en Acapulco de Juárez, teniendo una desvaloración de residencias de hasta de un 30 por ciento, según empresas inmobiliarias en la ciudad.

\section{ANTECEDENTES DE LA TEORÍA DE LOS PRECIOS HEDÓNICOS}

El análisis de los precios hedónicos se remonta desde el trabajo de Court (1939), a quien se le atribuye también como el inventor del término "hedónico". Él analiza índices de precios de automóviles con base en diferentes atributos, los cuales 
producen placer a los compradores. Durante los próximos 19 años siguientes no hubo un seguimiento sobre este enfoque; éste reapareció con los trabajos de Griliches: (Griliches, 1958), analizando precios de fertilizantes de acuerdo con diferentes componentes y (Griliches, 1961) donde analiza índices de precios de automóviles. Este último trabajo fue importante para reconocer a los precios hedónicos como una poderosa herramienta en econometría para predecir precios de bienes heterogéneos con base en sus diferentes componentes vistos como atributos o características. Sin embargo, fue Rosen (1974) quien formulara formalmente la teoría de los precios hedónicos como un problema en la economía del equilibrio espacial en la cual las decisiones tanto del consumidor como del comprador en el espacio de las características de un bien heterogéneo son guiadas por el conjunto de precios implícitos llamados precios hedónicos. La teoría de los precios hedónicos considera un bien en el mercado, que está compuesto por características o atributos y que es valorado por medio de éstos. Esta teoría trata de explicar el valor de un bien en función de aquellas características y así identifica la importancia relativa de cada una de ellas en el valor asignado del bien en el mercado. Aunque en la transacción del bien no existe un mercado directo de aquellas características, éstas tienen un precio implícito, llamado precio implícito marginal. Rosen, en su importante artículo (1974), define los precios hedónicos como los precios implícitos de los atributos que se revelan a los agentes económicos a partir de los precios observados de los productos diferenciados y las cantidades específicas de características asociadas a ellos.

Por medio de esta teoría es posible determinar estos precios implícitos en forma de disponibilidad a pagar por cada unidad de cambio de cada característica.

En su modelo hedónico, Rosen (1974) describe las acciones de los agentes económicos, comprador y vendedor, y condiciones para alcanzar un equilibrio en el mercado. Aquellas acciones permiten encontrar la relación de los precios del bien y sus características como una función, la cual es llamada función de precios hedónicos. En general, la forma de la función de precios hedónicos depende de las distribuciones de los consumidores y compradores. En aplicaciones empíricas, una vez elegida la forma de la función de precios hedónicos ésta se calcula usando regresión, llamada regresión hedónica, sobre los precios de mercado del bien dado en diferentes características.

Brevemente, el modelo de los precios hedónicos de Rosen (1974) se fundamenta teóricamente como sigue: se considera un mercado para bienes heterogéneos $Z$, es decir, $Z$ está compuesto por $n$ características o atributos $z_{1}, z_{2}, \ldots, z_{n}$, así que podemos escribirlo como un vector $n$-dimensional $Z=\left(z_{1}, z_{2}, \ldots, z_{n}\right)$. Cada $Z$ tiene un precio $P$ que depende de sus características, $P(Z)=\mathrm{P}\left(z_{1}, z_{2}, \ldots, z_{n}\right)$.

Se asume que hay un gran número de combinaciones de características para formar diferentes diseños del bien $Z$. El consumidor o comprador obtiene una 
utilidad $u\left(z_{1}, z_{2}, \ldots, z_{n}, x\right)$ por el consumo del bien $Z$ y de un bien compuesto $x$, y tiene una ingreso fijo $M$. Él desea maximizar su utilidad dado su ingreso $M$, para lo cual tiene que elegir un diseño $Z$ (una combinación de características) y un bien compuesto $x$, esto es:

$$
\operatorname{Max} u\left(z_{1}, z_{2}, \ldots, z_{n}, x\right)
$$

$$
\text { Sujeto a } P(Z)+x=M \text {. }
$$

Este problema requiere que se cumplan las condiciones de primer orden:

$$
\frac{u_{i}}{u_{x}}=P_{i}, \text { para } \mathrm{i}=1,2, \ldots, \mathrm{n},
$$

donde $u_{i}=\frac{\partial u}{\partial z_{-} i}, u_{x}=\frac{\partial u}{\partial x}$ y $P_{i}=\frac{\partial P}{\partial z_{\_} i}$. La derivada de $P$ respecto de la característica $Z_{i}$ es el precio hedónico de la característica $Z_{i}$.

Rosen define la cantidad que el consumidor está dispuesto a pagar por paquetes alternativos de características dado un nivel de utilidad y su ingreso $M$ como una función, llamada función de demanda $D(Z ; \mathcal{u}, M)$; formalmente la define de manera implícita $u=u(Z, M-D)$. Derivando implícitamente la función de demanda respecto de la característica $Z_{i}$ y considerando las condiciones de primer orden se obtiene que

$$
\frac{\partial D}{\partial z_{-} i}=P_{i} \text { para } \mathrm{i}=1,2, \ldots, \mathrm{n} .
$$

Así que la tasa a la cual el consumidor está dispuesto a pagar por la característica $Z_{i}$ es igual al precio hedónico de la característica $Z_{i}$. Entonces el diseño $Z$ donde la utilidad es maximizada es tal que para $\mathrm{i}=1,2, \ldots, \mathrm{n} D_{z_{i}}=P_{i}$ y $\mathrm{P}(\mathrm{Z})=D(Z$; $u, M)$, donde $P(Z)$ es el mínimo precio que el comprador debe pagar en el mercado por $Z$. Entonces en el óptimo $Z, P(Z)$ es tangente a $D(Z ; u, M)$.

Por otro lado, el otro agente en este mercado, el productor o vendedor, necesita maximizar su ganancia $\pi$ eligiendo un diseño $Z$ y el número $N(Z)$ de unidades producidas de este diseño, esto es:

$$
\operatorname{Max} \pi=P(Z) N-C(Z, N ; \beta)
$$


donde $C(Z, N)$ es el costo total de producir $N$ unidades del diseño $Z$ y $\beta$ representa tecnología especificada. Las condiciones requeridas de primer orden para este problema son:

$$
\begin{gathered}
P_{i}=\frac{C_{z_{-} i}}{N} \text { para } \mathrm{i}=1,2, \ldots, \mathrm{n}, \\
P(Z)=C_{N} .
\end{gathered}
$$

Esto nos dice que el precio hedónico de la característica $Z_{i}$ tiene que ser igual al costo total de producir una unidad de dicha característica; y el precio del diseño $Z$ debe ser igual al costo de producción de una unidad de este diseño. Simétricamente, Rosen define una función de oferta $O(Z ; \pi, \beta)$, la cual representa el precio unitario que el productor está dispuesto a aceptar por diseños del bien $Z$ con una ganancia constante cuando las cantidades producidas del diseño son óptimas. De la definición de la función de oferta se demuestra que

$$
\frac{\partial O}{O z \_i}=\frac{C_{z \_} i}{N}=\text { para } \mathrm{i}=1,2, \ldots, \mathrm{n} .
$$

Entonces la máxima ganancia $\pi$ y el diseño óptimo $Z$ deben satisfacer $\frac{\partial O}{O z_{-} i}=P_{i}$ para $\mathrm{i}=1,2, \ldots, \mathrm{n}$ y $P(Z)=O(Z ; \pi, \beta)$, donde $P(Z)$ es el máximo precio obtenido en el mercado por el diseño $Z$. Entonces en el óptimo $Z, P(Z)$ es tangente a $O(Z ; \pi, \beta)$. Por lo tanto, el equilibrio en el mercado hedónico se caracteriza por la tangencia de las curvas de demanda y oferta, dando origen a la función de precios hedónicos como la envolvente de tales funciones.

A priori no existe un criterio para elegir la forma funcional para la función de precios hedónicos, de hecho, Rosen (1974) establece que ésta en general no puede ser especificada a partir de consideraciones teóricas. A lo largo de la aplicación de esta teoría se han usado varias formas funcionales en el modelo econométrico para relacionar el precio de un bien heterogéneo y sus características.

Los bienes inmuebles, particularmente viviendas, son un ejemplo claro de un bien que está compuesto por una gran variedad de atributos o características.

En este artículo proponemos una forma funcional en el modelo con la cual obtenemos un buen ajuste a nuestros datos y la interpretación de los coeficientes estimados resulta sin dificultades.

Esta teoría ha tenido una gran aplicación para la valoración de atributos sobre viviendas, no sólo físicas sino también de localización, entorno y ambientales; por ejemplo, un primer estudio donde se aplica esta teoría a viviendas fue desarrollado por Ridker y Henning (1967), quienes estudian el efecto de la contaminación del aire sobre el precio de las viviendas en St. Louis, USA. Más tarde Sherwin Rosen (1974) propuso el método de dos pasos para determinar la relación empírica del 
precio y sus características. Otros trabajos, aplicando esta teoría en valores de la propiedad son: Thaler (1978), para la valoración del control de crimen en Rochester, New York; Witte, Sumka y Erekson (1979), para la valoración de la calidad de vivienda; Milon, Gressel y Mulkey (1984), para la valoración de amenidades relacionadas con agua; Hidano (2002), para la valoración del medio ambiente y políticas públicas; Troy y Grove (2008), para la valoración de crimen en Baltimore, MD; Santana y Núñez (2011), para la valuación de las preferencias por segregación en Bogotá.

Por otro lado, se ha demostrado que la teoría de los precios hedónicos está íntimamente relacionada con la teoría de transporte óptimo (Chiappori, McCann y Nesheim, 2010), la cual ha traído grandes avances no sólo en matemáticas sino en muchas áreas del conocimiento.

\section{LITERATURA DEL USO DE LA METODOLOGÍA DE PRECIOS HEDÓNICOS EN VIVIENDAS DE MÉXICO}

En México, la metodología de los precios hedónicos para la valuación de características de viviendas no ha sido ampliamente usada, a pesar de que uno de los primeros trabajos data desde hace más de tres décadas (Zorrilla, 1983). Este trabajo aplica esta metodología para el mercado de la vivienda en el Área Metropolitana de Monterrey (AMM), así como en (López, 2006), quien distingue entre el valor de la propiedad y el municipio de ubicación, y obtiene diferentes valuaciones de las características para cada análisis realizado.

En (Moreno, 2009) también se hace uso de la metodología de los precios hedónicos para el mercado de la vivienda en el AMM. Este autor toma en cuenta características de las viviendas que no son consideradas en trabajos anteriores, las que llama características de vecindarios, entre otras cosas, demuestra que la preferencia de los compradores por una vivienda también depende de cuestiones de tipo social como, por ejemplo, el nivel de educación y proporción de niños en el vecindario. Fitch, Soto y Garza (2013), estiman un modelo hedónico para la valoración de viviendas en San Nicolás de los Garza, México, considerando tres tipos de atributos de las viviendas: accesibilidad y transporte, aspectos socioeconómicos y externalidades ambientales. Ellos encuentran que los cambios en los atributos de externalidad sonora, grado promedio de escolaridad, densidad de habitantes por vivienda, cociente de localización entre transporte urbano-área y metros lineales del transporte urbano en el AGEB influyen en el valor de las viviendas. Otro de los estudios usando esta metodología es (Valdivia, 2014), la cual es aplicada a las viviendas nuevas ubicadas en la Zona Metropolitana del Valle de México. Este autor establece una estimación del precio de las viviendas 
nuevas en conjuntos o desarrollos habitacionales. Muestra que las características que más influyen en la determinación del precio de la vivienda son factores estructurales, pero también muestra que el precio de las viviendas se ha afectado negativamente por políticas públicas que favorecen la construcción de unidades habitacionales con problemas de accesibilidad. Sobrino (2014) explica el precio de las viviendas de la Ciudad de México a través de un modelo hedónico con variables estructurales, de externalidad de vecindad y accesibilidad, y demuestra que el mercado de vivienda en el Área Metropolitana de la Ciudad de México está dividido en submercados geográficos. Rodríguez Sánchez (2014) calcula la disponibilidad a pagar de los compradores de viviendas en México por reducir la contaminación del aire usando un enfoque hedónico, pero incorporando costos de movilidad, obteniendo así un modelo de clasificación residencial. Monkkonen (2016) estudia la variación en el valor del título de propiedad en un gran número de ciudades de México. Los resultados de modelos hedónicos demuestran que el valor de la vivienda se incrementa si se tiene un título de propiedad de ésta en ciudades con una población más educada, más activamente involucrada en el proceso político y donde hay más competencia política. Recientemente, en (Pulido, Estrada, Zentella y Guevara, 2017) la metodología de los precios hedónicos es aplicada para probar que la expansión urbana en la Zona Metropolitana del Valle de México tiene costos sociales superiores a un crecimiento menos extensivo de la ciudad. Los resultados del modelo demuestran, por ejemplo, que a mayor distancia respecto del centro de transporte más cercano, el precio de la vivienda disminuye, y a mayor velocidad promedio hacia el centro de transporte más cercano el precio de la vivienda aumenta. Con ello los autores pueden demostrar que la pérdida de ingreso para una persona que habita lejos de un centro de transporte es mayor que la reducción en el precio de compra de dicha casa periférica.

Hasta donde sabemos, el trabajo de Núñez, Paredes, Garduño-Rivera (2017) es de los primeros que incluye una variable para medir efectos de violencia sobre viviendas en México, usando el enfoque del modelo de los precios hedónicos; esta variable es la tasa de crimen por municipio. Los autores calculan la disponibilidad marginal de pagar por crimen y otras amenidades en México. Demuestran que el salario es afectado negativamente por el crimen, mientras que para algunos estados del país el crimen resulta ser una amenidad; la explicación que dan los autores de este último resultado es que tal vez se deba a que algunos de los más antiguos carteles de droga se encuentran en dichos estados. Respecto a otras características prueban, por ejemplo, que altos niveles de contaminación afectan negativamente los salarios y los precios de la renta de viviendas. 


\section{DATOS Y VARIABLES EN EL MODELO HEDÓNICO}

Los precios implícitos de las características del bien se obtienen por medio de la estimación de los coeficientes de las variables que corresponden a estas características en la función de precios especificada, por medio de una regresión, la cual es llamada regresión hedónica. Para esto construimos una base de datos de precios de viviendas observadas con diferentes características.

Nuestra base de datos consiste en 184 viviendas ubicadas a lo largo y ancho de la ciudad, las cuales fueron ofertadas y capturadas en la página web de inmobiliarias en Acapulco en el mes de diciembre de 2016. Las variables que corresponden a las características físicas de viviendas consideradas en nuestro estudio son las siguientes:

(1) $C=$ Variable dicotómica que toma el valor uno si la vivienda es una casa y cero en otro caso.

(2) $C C=$ Variable dicotómica que toma el valor uno si la vivienda está en un condominio y cero en otro caso.

(3) $N R=$ Número de recámaras de la vivienda.

(4) $N B=$ Número de baños de la vivienda.

(5) $N E=$ Número de estacionamientos de la vivienda.

(6) $A=$ Número de años de la vivienda.

(7) $S=$ Área total de la vivienda (en metros cuadrados).

(8) $T C=$ Área total construida de la vivienda (en metros cuadrados).

Las viviendas se clasifican en casa, casa en condominio y departamento, y este último funge como la categoría de referencia en el modelo. Los datos correspondientes a estas características fueron capturados al mismo tiempo que se capturó el precio de la vivienda.

Las variables que corresponden a las características de localización son las siguientes:

(9) $D P=$ Distancia en ruta a la playa más cercana (en metros).

(10) $L=$ Variable dicotómica que toma el valor uno si la casa está localizada en una zona privada con seguridad permanente y vista a la playa.

(11) $D R=$ Distancia en ruta a la colonia Ciudad Renacimiento (en metros).

Más precisamente, $D P$ es la distancia en ruta del centroide de la colonia donde se encuentra ubicada la vivienda a la avenida más cercana con acceso a la playa y $D R$ es la distancia en ruta del centroide de la colonia Ciudad Renacimiento al 
centroide de la colonia donde se ubica la vivienda observada. Estas distancias fueron obtenidas usando Google Earth.

Finalmente, la variable dependiente es:

$P=$ Precio de la vivienda (en MXN).

Las estadísticas descriptivas de las variables (1)-(11) se muestran en la tabla 1.

Tabla 1. Estadisticas descriptivas.

\begin{tabular}{|c|c|c|c|c|}
\hline Variable & Media & Máx. & Mín. & Desviación estándar \\
\hline Precio & 4585936 & 40000000 & 360000 & 6294015.8 \\
\hline C & 0.337 & 1 & 0 & 0.4739595 \\
\hline$C C$ & 0.4565 & 1 & 0 & 0.4994651 \\
\hline$N R$ & 3.565 & 10 & 1 & 1.3207412 \\
\hline$N B$ & 3.505 & 12 & 1 & 1.9333068 \\
\hline$S$ & 437.3 & 23000 & 5 & 1705.9942 \\
\hline$T C$ & 285.5 & 2812 & 48 & 320.17785 \\
\hline$N E$ & 1.902 & 22 & 0 & 2.1906896 \\
\hline$A$ & 7.31 & 40 & 0 & 8.9195991 \\
\hline$D P$ & 2907 & 11300 & 78 & 2863.3492 \\
\hline$L$ & 0.08696 & 1 & 0 & 0.282540 \\
\hline$D R$ & 11138 & 23500 & 0 & 5669.9349 \\
\hline Núm. de observacio & & & & \\
\hline
\end{tabular}

La tabla 1 muestra que, en promedio, las viviendas en Acapulco de Juárez tienen un precio de aproximadamente cuatro millones y medio. De esta muestra, el 33 por ciento son casas, el 45 por ciento son viviendas en condominios y el resto son departamentos. Estas viviendas en promedio tienen tres recámaras y tres baños, con 437.3 metros cuadrados de superficie total y 285.5 metros cuadrados de área construida. Además, en promedio tienen dos estacionamientos y una vida de construcción de siete años. La distancia promedio en ruta de las viviendas a la playa más cercana es de 2,907 metros. Aproximadamente un nueve por ciento de las viviendas se localiza en zona privada con seguridad permanente y vista a la playa. Finalmente, la distancia promedio en ruta de las viviendas a la colonia Ciudad Renacimiento es de 11,138 metros. 


\section{FORMA FUNCIONAL Y ESTIMACIONES}

En econometría se ha usado bastante la transformación Box-Cox (Box y Cox, 1964) para la forma funcional hedónica de precios, la cual está dada por:

$$
P^{\lambda \_1}=\beta_{0}+\sum_{j=1}^{N} \beta_{j} X_{j}^{\lambda \_2}+\varepsilon
$$

donde $\varepsilon$ es el término de error, $N$ es el número de variables explicativas e independientes en la regresión y la variable dependientes y las independientes son transformadas en $P^{\lambda_{-} l}$ y en $X_{j}^{\lambda_{-}{ }^{2}}$, respectivamente, como sigue:

$$
y^{\lambda}=\left\{\begin{array}{cc}
\frac{y^{\lambda}-1}{\lambda}, & \lambda \neq 0, y>0 \\
\ln (y), & \lambda=0, y>0
\end{array}\right.
$$

Esta transformación contiene casos especiales que se han aplicado en estudios de valuación hedónica como, por ejemplo: cuando $\lambda_{1}=\lambda_{2}$, obteniendo un modelo lineal, para $\lambda_{1}=0$ y $\lambda_{2}=1$ obteniendo un modelo semilogarítmico y para $\lambda_{1}=0=\lambda_{2}$, obtenemos el modelo logarítmico.

El precio hedónico marginal para el atributo o característica $X_{j}$ está dado por:

$$
\frac{\partial P}{\partial X_{j}}=\beta_{j} P^{1-\lambda_{1}} X_{j}^{\lambda_{2}-1}
$$

La ecuación que es estimada en la regresión de nuestros datos para obtener los precios implícitos de las características que contempla nuestro estudio es la siguiente:

$$
\begin{gathered}
\ln (P)=\beta_{0}+\beta_{1} C+\beta_{2} C C+\beta_{3} \ln (N R)+\beta_{4} \ln (N B)+\beta_{5} \ln (S)+\beta_{6} \ln (T C) \\
+\beta_{7} N E+\beta_{8} A+\beta_{9} D P+\beta_{10} L+\beta_{11} D R
\end{gathered}
$$

Esta forma funcional hedónica corresponde a un modelo generalizado de la transformación Box-Cox, debido a que para algunas variables explicativas $\lambda_{2}$ toma el valor 0 y en otras el valor 1 . Este modelo se ha propuesto después de haber ensayado con otros: como el lineal, el semilogarítimico, el logarítimico (sin transformar las variables dicotómicas y $D R$, ya que toman el valor cero). 
Los modelos ensayados resultaron un mal ajuste o no cumplieron con alguna de las tres hipótesis del análisis de regresión. En cambio, el modelo dado en (1) supera estos problemas. Debido a su simplicidad se tiene una interpretación de los coeficientes sin dificultades. A continuación, describimos el modelo propuesto y damos sus propiedades, las cuales nos llevaron a su elección para nuestro estudio.

Se usó el método de mínimos cuadrados ordinarios para las estimaciones de los coeficientes $\beta_{j}$ y usamos el software R para tales estimaciones. Además, con este software realizamos las pruebas de hipótesis en el análisis de regresión.

En la tabla 2 presentamos los coeficientes estimados para la función de precios dada en (1), los errores estándar y el p-valor para cada una de las variables.

Notemos que todas las variables resultaron estadísticamente significativas, excepto las variables, $N E, A$ y $\ln (N R)$. Obtuvimos un buen ajuste, dado que el valor del coeficiente ajustado de determinación de $R_{\alpha}^{2}=0.8695$, el cual es mayor a algunos presentados en trabajos reportados, como, por ejemplo, el obtenido por Fitch, Soto y Garza (2013).

Tabla 2. Estimación de coeficientes del modelo hedónico (1).

\begin{tabular}{|c|c|c|c|}
\hline Variable & Coeficiente & Error estándar & p-valor \\
\hline Intercept & $9.782 \mathrm{e}+00$ & $3.716 \mathrm{e}-01$ & $<2 \mathrm{e}-16$ \\
\hline C & $-6.168 e-01$ & $1.002 \mathrm{e}-01$ & $5.07 e-09$ \\
\hline$C C$ & $-3.365 e-01$ & $8.268 \mathrm{e}-02$ & 7.17e-05 \\
\hline $\ln (N R)$ & $2.880 e-01$ & $1.537 \mathrm{e}-01$ & 0.062661 \\
\hline $\ln (N B)$ & $3.457 e-01$ & $1.008 \mathrm{e}-01$ & 0.000757 \\
\hline $\ln (S)$ & $2.494 \mathrm{e}-01$ & $6.018 \mathrm{e}-02$ & $5.36 \mathrm{e}-05$ \\
\hline $\ln (T C)$ & $7.203 e-01$ & $9.358 \mathrm{e}-02$ & $1.04 \mathrm{e}-12$ \\
\hline$N E$ & $-3.106 e-03$ & $1.908 \mathrm{e}-02$ & 0.870844 \\
\hline$A$ & $-4.269 e-03$ & $3.439 \mathrm{e}-03$ & 0.216159 \\
\hline$D P$ & $-5.415 e-05$ & $1.611 \mathrm{e}-05$ & 0.000954 \\
\hline$L$ & $4.378 \mathrm{e}-01$ & $1.189 \mathrm{e}-01$ & 0.000311 \\
\hline$D R$ & $1.972 \mathrm{e}-05$ & $7.942 \mathrm{e}-06$ & 0.014001 \\
\hline \multicolumn{4}{|c|}{$R_{a}^{2}=0.8695$} \\
\hline
\end{tabular}

Mediante el análisis VIF estudiamos cuestiones de colinealidad para este modelo ajustado. Todas las variables resultaron con un VIF menor al VIF global del modelo ajustado, lo cual nos da indicios de no-colinealidad. Los valores se muestran en la tabla 3. 
Tabla 3. Valores de los VIF.

\begin{tabular}{cc}
\hline Variable & VIF \\
\hline$C$ & 2.537945 \\
$C C$ & 1.919776 \\
$\ln (N R)$ & 3.268803 \\
$\ln (N B)$ & 3.354335 \\
$\ln (S)$ & 3.729917 \\
$\ln (T C)$ & 5.782719 \\
$N E$ & 1.965948 \\
$A$ & 1.059150 \\
$D P$ & 2.395056 \\
$L$ & 1.271300 \\
$D R$ & 2.282842 \\
\hline \multicolumn{2}{c}{} \\
\hline
\end{tabular}

Las hipótesis bajo las cuales se rige el modelo de regresión lineal, fueron verificadas. Por medio del test Breusch-Pagan encontramos no evidencia de heterocedasticidad, a un nivel de confianza del 99 por ciento con un valor del estadístico BP de 26.646 y 11 grados de libertad. La normalidad de los errores la verificamos usando el test Shapiro-Wilk a un nivel de confianza del 99 por ciento con el estadístico $\mathrm{W}=0.97492$ y número de observaciones $n=184$; y finalmente, la no evidencia de correlación de los errores se verificó mediante el test Durbin-Watson a un nivel de confianza del 95 por ciento.

Para la validación de nuestro modelo usamos la validación cruzada una a una, la cual consiste en ajustar el modelo para cada observación i con las $n-1$ observaciones restantes y con él predecir la respuesta $i, \hat{y}_{i}^{(i)}$. Así que la robustez de nuestro modelo la evaluamos por medio del coeficiente de robustez dado por:

$$
B^{2}=\frac{\sum_{i=1}^{n}\left(y_{1}-\hat{y}_{i}\right)^{2}}{\sum_{i=1}^{n}\left(y_{1}-\hat{y}_{i}^{(i)}\right)^{2}},
$$

donde $\hat{y}_{i}$ es el valor predecido de la i-ésima observación usando el total de datos en el ajuste y $\hat{y}_{i}^{(i)}$ es la i-ésima observación predicha con los $n$ - 1 datos en el ajuste obtenidos por eliminar la i-ésima observación. Este coeficiente es un valor entre 0 y 1 , un valor cercano a 1 significa que todos los valores predecidos $\hat{y}_{i}^{(i)}$ son cercanos a $\hat{y}_{i}$, y si es cercano a cero entonces habrá mucha diferencia entre aquellos valores. Nosotros hemos obtenido un valor de $B^{2}=0.83$, por lo cual consideramos nuestro modelo robusto. 


\section{INTERPRETACIÓN DE LOS RESULTADOS}

Una vez verificado que nuestro modelo es un buen ajuste a nuestros datos, que cumple con las hipótesis del análisis de regresión y es robusto, continuamos con la interpretación de los resultados.

La variable $C$ es significativa al nivel del 99.9 por ciento de confianza y el signo negativo de su coeficiente nos indica que el precio de una casa es menor que el precio de un departamento, ceteris paribus. En efecto, sea $\beta_{\mathcal{C}}=-0.6168$, el coeficiente estimado para $C$, la relación de los precios de una casa y un departamento está dada por $P_{\text {casa }}=e^{\beta_{C}} P_{\text {depto }}=0.5396 P_{\text {depto }}$. Por ejemplo, si un departamento cuesta 5000,000 mxn, entonces una casa con iguales características tendrá un precio de 2698,000 mxn. La variable CC también resultó significativa al nivel de confianza del 99.9 por ciento y con coeficiente negativo, indicando que el precio de una casa en condominio tiene un precio menor que el de un departamento, manteniendo las otras variables constantes.

La relación de precios se obtiene de manera similar como la variable $C$. Tenemos entonces que $P_{\text {casaencondo }}=e^{\beta_{c c}} P_{\text {depto }}=0.7142 P_{\text {depto }}$. Ejemplo, si un departamento tiene un precio de $5000,000 \mathrm{mxn}$, el precio de una casa en condominio con iguales características tendrá un precio de 3571,000 mxn.

La variable $\ln (N R)$ no resultó ser significativa, mientras que la variable $\ln (\mathrm{NB})$ sí, a un nivel de confianza del 99.9 por ciento y además con signo esperado, positivo. Su coeficiente 0.3457 es interpretado como una elasticidad, indicando que por cada 1 por ciento de incremento de $N B$, hay un 0.3457 por ciento de incremento en el valor de la vivienda.

Otra variable física que resultó significativa es $\ln (S)$ al mismo nivel que las anteriores y con signo positivo, el cual indica que conforme el área de una vivienda aumente, el precio de la vivienda incrementa. Más precisamente, por cada 1 por ciento de incremento de área de la vivienda, hay un incremento del 0.2494 por ciento en el valor. La variable $\ln (T C)$ es significativa al nivel de confianza del 99.9 por ciento con signo positivo, y su coeficiente se interpreta como una elasticidad, como la variable $\ln (S)$.

En cuanto a las variables de localización, todas resultaron significativas, por ejemplo, la distancia a la playa $(D P)$, es significativa al nivel de confianza del 99.9 por ciento y su coeficiente con signo esperado, negativo. Su valor -0.00005415 nos indica que por cada metro que la vivienda se aleje de la playa más cercana, su valor decrece en 0.005415 por ciento. Por ejemplo, si el valor de la vivienda es de $1000,000 \mathrm{mxn}$, entonces decrece en $54.15 \mathrm{mxn}$ por cada metro que la vivienda se aleje de la playa más cercana. La figura 3 muestra el ajuste lineal entre $\ln (P)$ y $D P$. 
Figura 3. Relación lineal entre In P y DP.

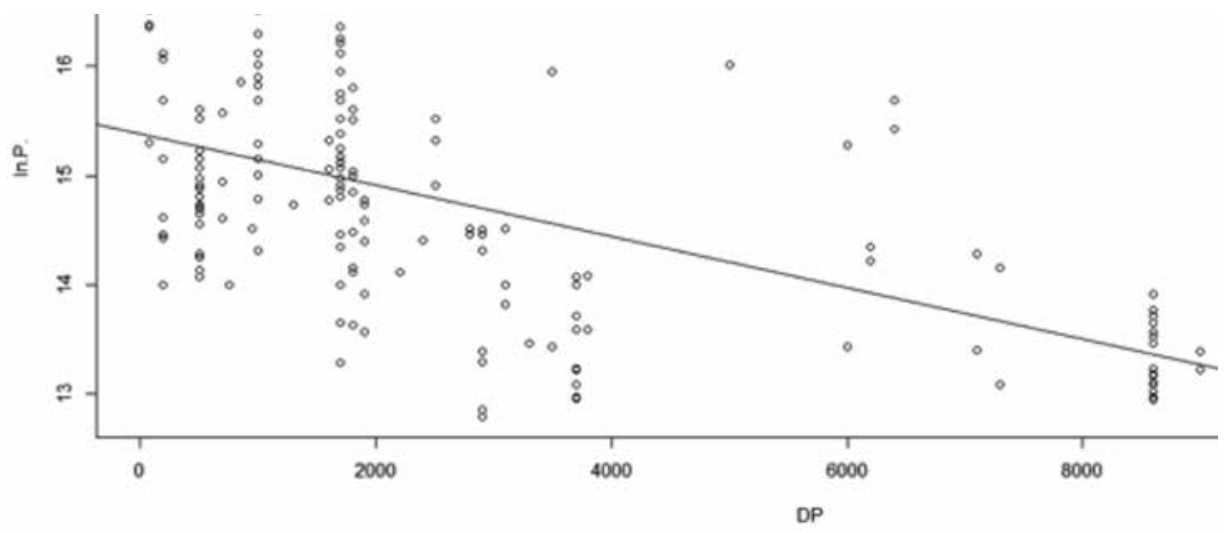

La variable dicotómica $L$, también resultó significativa al nivel del 99.9 por ciento y con signo esperado, positivo, indicando que una vivienda dentro de esa zona privada descrita anteriormente, tiene un precio mayor al de una vivienda ubicada fuera de aquella zona, ceteris paribus. La relación de precios se obtiene como las variables dicotómicas anteriores.

Finalmente, acerca de nuestra variable de mayor interés, la distancia a la colonia Ciudad Renacimiento resultó significativa al nivel de confianza del 95 por ciento con signo esperado. Su coeficiente 0.00001972 indica que por cada metro que la vivienda se aleje de aquella zona, su valor incrementa en 0.001972 por ciento. Así, por ejemplo, si la vivienda tiene un precio de 1000,000 mxn, entonces el comprador estará dispuesto a pagar $19.72 \mathrm{mxn}$ por cada metro que ésta se aleje de aquella zona. Entonces podemos atribuir una pérdida de valor de la vivienda cuando se vive cerca de esta colonia, la cual es la más insegura de Acapulco de Juárez; probando así nuestra hipótesis planteada. El ajuste lineal de $D R$ contra $\ln (P)$ se muestra en la figura 4 . 
Figura 4. Relación lineal entre In $P$ y $D R$.

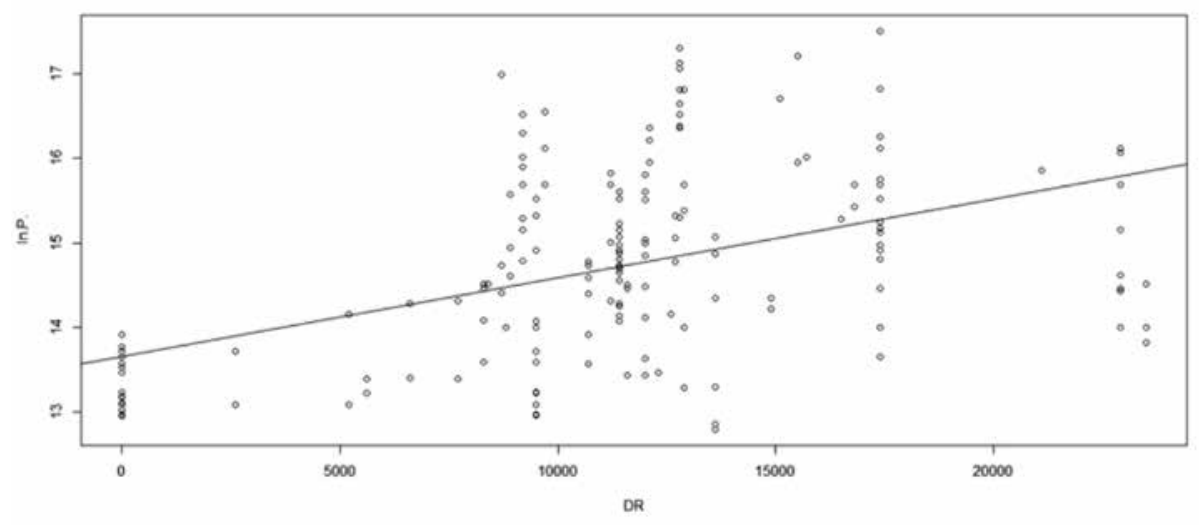

\section{CONCLUSIONES}

En este artículo hemos ajustado un modelo hedónico para conocer los efectos de la inseguridad en Acapulco de Juárez en el precio de las viviendas de esta ciudad, usando la distancia a la colonia más insegura de la ciudad: Ciudad Renacimiento. Este trabajo contribuye a la literatura sobre la valoración de violencia en precios de viviendas en México a través de un modelo hedónico, que de hecho es bastante pobre.

Nuestra mayor contribución en este artículo es probar que existe un efecto negativo de la inseguridad sobre el precio de las viviendas de Acapulco de Juárez, obteniendo que el valor de las viviendas decrece con la cercanía a dicha colonia insegura. Nuestros resultados corroboran la hipótesis planteada en este trabajo. Además, hemos cuantificado el valor por el incremento por cada metro que la vivienda se aleje de aquella colonia, que resultó un incremento del 0.001972 por ciento de su precio, atendiendo a una de nuestras preguntas de investigación.

De las características de las viviendas consideradas en nuestro modelo hedónico, la mayor parte resultaron significativas, y las variables que explican con mayor nivel de significancia (99.9 por ciento) el precio de las viviendas en Acapulco de Juárez son las de tipo de vivienda, algunas estructurales: $N B, S$ y $T C$, en su forma logarítmica; y de localización: $D P$ y $L$, respondiendo a la primera pregunta de investigación. Respecto a nuestra tercera pregunta de investigación, nuestro modelo nos indica que el comprador estaría dispuesto a pagar más por la cercanía a la playa que por la lejanía a la colonia más insegura. Lo que sugiere que a pesar de que el efecto de la inseguridad es importante para el precio de las 
viviendas, para el comprador es más importante la amenidad de vivir cerca de la playa.

Una necesidad que el presente trabajo plantea, es incorporar otras variables que cuantifiquen de mejor manera el efecto de la inseguridad en los precios de vivienda en la ciudad, por ejemplo, se podría considerar el nivel de incidencia en homicidios dolosos, robo de vehículos y armamento por colonias, sin embargo, esta información es reservada debido a la delicada situación en la que se encuentra el puerto de Acapulco; de hecho, la obtención de datos es muchas veces un problema común a la que se tiene que enfrentar en este tipo de modelos. Pese a esto, nuestro modelo predice precios implícitos razonables y consistentes. Un futuro trabajo a desarrollar sería explorar e incorporar otras variables en un modelo hedónico para saber si los efectos de la cercanía a la playa en el valor de las viviendas en Acapulco están condicionados por los niveles de violencia en dicha ciudad.

\section{REFERENCIAS BIBLIOGRÁFICAS}

Amérigo, M. y Pérez-López, R. (2010), “Ambientes residenciales”, en Aragones, J. I. y Amérigo, M. (eds.), Psicología ambiental, 3a. ed., pp. 59-75.

Área Geoestadística Municipal 120010001 (2010), <http://geoweb.inegi.org.mx/ AHL/realizaBusquedaurl.do?cvegeo $=120010001>(15$ de octubre de 2016$)$.

Baranzini, A.; Ramírez, J.; Schaerer, C. y Thalmann, P. (2008), "Hedonics methods in housing markets", Pricing environmental amenities and segregation, Springer.

Box, G. E. y Cox, D. R. (1964), "An analysis transformations", Journal of the Royal Statistical Society, Series B (Methodological), vol. 26, núm. 2, pp. 211-252.

Canter, D. (1977), Psicología del lugar, Editorial Concepto, México.

Chiappori, P. A.; McCann, R. J. y Nesheim, L. P. (2010), "Hedonic Price Equilibria, Stable Matching, and Optimal Transport: Equivalence, Topology, and Uniqueness", Economic Theory, vol. 42, núm.2, pp. 317-354.

Court, A. T. (1939), "Hedonic price indexes with automotive examples", in The Dynamics of Automobile Demanda, General Motors, Nueva York, pp. 98-119.

Enciclopedia Guerrerense (2012), <http://www.enciclopediagro.org/index.php/ atlas-municipal/resumen-municipal/1703-acapulco-de-juarez $>$ (18 de enero de 2017).

El Universal (2017), <http://www.eluniversal.com.mx/articulo/nacion $\backslash \backslash /$ seguridad/2017/01/13/acapulco-el-municipio-mas-violento-del-pais-cns $>$ (28 de enero de 2017). 
Fitch, J. M.; Soto, K. y Garza, R. (2013), "Valuación de la calidad urbano-ambiental. Una modelación hedónica: San Nicolás de los Garza, México”, Estudios Demográficos y Urbanos, vol. 28, núm. 2 (83), pp. 383-428.

Griliches, Z. (1958), "The demand for fertilizer: 'An econometric reinterpretation of a technical change"', Journal of Farm Economics, vol. 40, pp. 591-606. (1961), "Hedonic prices for automobiles: An econometric analysis of quality change", The Price Statistics of the Federal Government, General Series, núm. 73, Columbia University Press for the National Bureau of Economic Research, Nueva York, pp. 137-196.

Hidano, Noboru (2002), The economic valuation of the environment and public policy. A hedonic approach. New horizons in environmental economics Ser, Edward Elgar Publishing, Inc. Northampton, Massachusetts.

INEGI (2010), Instituto Nacional de Estadística y Geografía. Censo de Población y Vivienda 2010. <http://www.inegi.org.mx/est/contenidos/proyectos/ccpv/ cpv2010/\Default.aspx> (12 de noviembre de 2016).

López Rizzo, H. C. (2006), Valuación de las características de la vivienda del AMM mediante la metodología de precios hedónicos, tesis de maestría en Economía, Monterrey, UANL, Facultad de Economía.

López Velasco, R.; Rodríguez, A.; Barragán, C.; Castellanos, C.; Palacios, R. y Martínez, M. (2012), "Tourism and Environmental Pollution in the Urban Periphery of Acapulco: Ciudad Renacimiento", Revista El Periplo Sustentable, núm.23, pp. 113-141.

Milon, Walter; Gressel, Jonathan y Mulkey, David (1984), "Hedonic Amenity Valuation and Functional Form Specification", Land Economics, vol. 60, núm. 4, pp. 378-387.

Monkkonen, P. (2016), "Where do property rights matter more? Explaining the variation in demand for property titles across cities in Mexico", World Development, vol. 88, pp. 67-78.

Moreno Murrieta, R. E. (2009), Características de la vivienda ideal: Una valoración a través de la metodología de precios hedónicos, tesis de maestría en Economía, Monterrey, UANL, Facultad de Economía.

Nuñez, H. M.; Paredes, D. y Garduño-Rivera, R. (2017), "Is crime in Mexico a disamenity? Evidence from a hedonic valuation approach", The Annals of Regional Science, vol. 59, núm. 1, pp. 171-187.

Pulido, L.; Alberto, J.; Estrada Díaz, G.; Zentella Gómez, J. C. y Guevara Sanginés, A. (2017), "Los costos de la expansión urbana: aproximación a partir de un modelo de precios hedónicos en la Zona Metropolitana del Valle de México", Estudios Demográficos y Urbanos, vol. 32, núm. 1, pp. 37-63. 
Ridker, R. G. y Henning, J. A. (1967), "The determinants of residential property values with special reference to air pollution", The Review of Economics and Statistics, vol. 49, núm. 2, pp. 246-257.

Rodríguez Sánchez, J. I. (2014), “Do Mexicans care about air population?”, Lat Am Econ Rev, vol. 23, núm. 9, pp. 1-24.

Rosen, Sherwin (1974), "Hedonic Prices and Implicit Markets: Product Differentiation in Pure Competition”, Journal of Political Economy, vol. 82, núm. 1, pp. 34-55. Santana, L. y Núñez, L. (2011), "Una aproximación hedónica al efecto de las preferencias por segregación en el precio del suelo urbano en Bogotá", Equidad y Desarrollo, núm.16, pp. 139-162.

Seguridad, Justicia y Paz (2015), Consejo Ciudadano para la Seguridad Pública y Justicia Penal A.C. <https://www.seguridadjusticiaypaz.org.mx/biblioteca/ download/6-prensa/239-las-50-ciudades-mas-violentas-del-mundo-2016-metodologia $>$ (15 de diciembre de 2015). (2016), Consejo Ciudadano para la Seguridad Pública y Justicia Penal A.C. $<$ http://www.seguridadjusticiaypaz.org.mx/sala-de-prensa/1356> (30 de enero de 2017).

Sobrino, Jaime (2014), "Housing prices and submarkets in Mexico City: A hedonic assessment", Estudios Económicos, vol. 29, núm. 1, pp. 57-84.

Thaler, Richard (1978), "A note on the value of crime control: Evidence from the property market", Journal of Urban Economics, vol. 5, núm. 1, pp. 137-145.

Troy, A. J. y Grove, Morgan (2008), "Property values, parks, and crime: A hedonic analysis in Baltimore", Landscape and Urban Planning, vol. 87, núm.3, pp. 233-245.

Valdivia, R. (2014), Modelos de precios hedónicos para la vivienda nueva en la ZMVM. Estudio de caso en la Zona Metropolitana del Valle de México.

Witte, A. D.; Sumka, Howard J. y Erekson, H. (1979), "An Estimate of a Structural Hedonic Price Model of The Housing Market: An Application of Rosen's Theory of Implicit Markets", Econometrica, vol. 47, núm.5, pp. 1151-1173.

World Tourism Organization (2015), "International Tourism Receipts", in UnWTO Tourism Highlights, 2015 Edition. <http://www.e-unwto.org/doi/ pdf/10.18111/9789284416899> (9 de enero de 2017).

Zorrilla Bustamante, A. (1983), Precios implícitos de las características de la vivienda en el $A M M$, tesis de licenciatura en Economía, Monterrey, UANL, Facultad de Economía. 
